\title{
A PROMOÇÃO DA SAÚdE NAS PRÁTICAS EDUCATIVAS DA SAÚDE DA FAMÍLIA
}

Pamela Camila Fernandes Rumor ${ }^{1}$, Isabel Berns ${ }^{2}$, Ivonete Teresinha Schülter Buss Heidemann ${ }^{3}$, Larissa Helena Lamego Mattos ${ }^{4}$, Antônio Miranda Wosny

RESUMO: Pesquisa qualitativa com o objetivo de identificar e analisar como as principais ações de Promoção da Saúde - estabelecidas pela Carta de Ottawa - estão sendo trabalhadas nas práticas educativas de equipes de Saúde da Família de um Centro de Saúde do Município de Florianópolis, Santa Catarina. Para a coleta e análise dos dados foi utilizado o itinerário de pesquisa de Paulo Freire, em 16 círculos de cultura realizados com usuários e profissionais. Os resultados apontaram que a incorporação dos princípios da Carta de Ottawa permanece distante da prática concreta dos profissionais, especialmente devido à sustentação do modelo biomédico, predominante no setor saúde. Salienta-se a importância da atuação das equipes numa perspectiva interdisciplinar e dialógica, que estimule a participação do usuário para que, em conjunto com os profissionais, construam uma prática de saúde mais emancipatória e autônoma sobre os determinantes do processo saúde e doença.

PALAVRAS-CHAVE: Promoção da saúde; Educação em saúde; Saúde da família.

\section{HEALTH PROMOTION IN EDUCATIONAL PRACTICES OF FAMILY HEALTH}

\begin{abstract}
A qualitative study aiming to identify and analyze how the key actions for Health Promotion - established by the Ottawa Charter - were developed in the educational practices of the Family Health teams in a health center in the city of Florianopolis, Santa Catarina. Paulo Freire's route search was used for data collection and analysis 16 circles of culture, both with professionals and users. The results showed that the incorporation of the principles of Ottawa Charter remains far from the actual practice of professionals, especially due to the biomedical model, which is prevalent in the health sector. We emphasize the importance of an interdisciplinary and dialogical team work, in order to encourage the participation of the user so that, together with professionals, they could build a more emancipated and autonomous health practice on the determinants of health and illness process.
\end{abstract}

KEYWORDS: health promotion; Health education; Family health.

\section{PROMOCIÓN DE LA SALUD EN LAS PRÁCTICAS EDUCATIVAS DE LA SALUD DE LA FAMILIA}

RESUMEN: Investigación cualitativa con el objetivo de identificar y analizar como las principales acciones de Promoción de la Salud - establecidas por la Carta de Ottawa - son trabajadas en las prácticas educativas de equipos de Salud de la Familia de un Centro de Salud del municipio de Florianópolis, Santa Catarina. Para recoger y analizar los datos fue utilizado el itinerario de investigación de Paulo Freire en 16 círculos de cultura, realizados con usuarios y profesionales. Los resultados apuntaron que la incorporación de los principios de la Carta de Ottawa permanece lejos de la práctica concreta de los profesionales, especialmente a causa de la sustentación del modelo biomédico, predominante en sector de salud. Se resalta la importancia de la actuación de los equipos en una perspectiva interdisciplinar y dialógica, que estimule la participación del usuario, a fin de que, en conjunto con los profesionales, construyan una práctica de salud que emancipe y sea autónoma acerca de los determinantes del proceso salud y enfermedad.

PALABRAS CLAVE: Promoción de la salud; Educación en salud; Salud de la familia.

\footnotetext{
${ }^{1}$ Enfermeira. Mestranda do Programa de Pós-Graduação em Enfermagem da Universidade Federal de Santa Catarina-PEN/UFSC. Bolsista CAPES. Membro do Núcleo de Extensão e Pesquisa em Enfermagem e Promoção da Saúde-NEPEPS/UFSC.

${ }^{2}$ Enfermeira Assistencial do Hospital Universitário da UFSC. Especializanda em Saúde da Família pelo Departamento de Saúde Pública da UFSC. Membro do NEPEPS.

${ }^{3}$ Enfermeira. Doutor em Enfermagem. Professor do Departamento de Enfermagem e do PEN/UFSC. Vice-Coordenadora do NEPEPS. ${ }^{4}$ Enfermeira da Secretaria de Saúde da Prefeitura Municipal de Florianópolis/SC. Especialista em Saúde Pública. Membro do NEPEPS. ${ }^{5}$ Enfermeiro. Doutor em Enfermagem. Professor do Departamento de Enfermagem da UFSC. Membro do NEPEPS.
}

\section{Autor correspondente:}

Pamela Camila Fernandes Rumor

Universidade Federal de Santa Catarina

R. Padre Raulino Reitz, 365 - 88113-120 - São José-SC-Brasil.

Recebido: 05/04/10

E-mail: pamrumor@hotmail.com Aprovado: 20/11/10 


\section{INTRODUÇÃO}

O conceito moderno de Promoção da Saúde vem sendo discutido e abordado mais intensamente nos últimos 30 anos como uma proposta internacional para lutar pelos múltiplos problemas de saúde que afetam a população. Entendida de uma forma ampliada e não somente como um momento anterior à doença, a Promoção da Saúde foi definida pela Carta de Ottawa como sendo um processo de empoderamento de indivíduos e comunidades sobre os determinantes de saúde, possibilitando conquistas em modos e condições de vida ${ }^{(1)}$.

No âmbito do Sistema Único de Saúde (SUS), vivenciamos um processo de mudança do modelo assistencial às doenças, para um modelo de promoção social de saúde que adota como estratégia fundamental a concretização de ações de Promoção da Saúde, de acordo com sua concepção. Por integrar o SUS, a Estratégia de Saúde da Família (ESF) coincide com os objetivos da Promoção da Saúde, fundamenta-se nos princípios de integralidade da atenção à saúde e da participação popular ${ }^{(2-3)}$, e tem como foco de ação o indivíduo, a família e a coletividade.

A educação em saúde pode ser considerada como conteúdo naturalmente integrante em qualquer nível da atenção. Neste sentido, a educação não é só aquela planejada pelas equipes como programas ou atividades educativas da ESF. Faz parte de qualquer ação de saúde tanto de promoção, prevenção como de cura e reabilitação. Isso depende exclusivamente da atitude educativa do profissional ou da equipe. A educação em saúde enfatiza a prevenção de doenças e continua sendo vista, no terreno da saúde pública, de maneira individual. Esta abordagem representa a saúde, predominantemente, como uma questão de responsabilidade pessoal e indicação do "bom" uso da autonomia individual. Ela é conceituada como resultado de tomada de decisão racional quanto ao comportamento pessoal, o que pressupõe que o conhecimento esteja diretamente relacionado à mudança de comportamento. Tal lógica tem sido aplicada a muitos dos problemas de saúde pública na atualidade, vistos como solucionáveis por meio do ensino de quais comportamentos são adequados para a manutenção do "natural e original" estado de saúde de todos ${ }^{(4)}$.

A Promoção da Saúde, na prática das ações de educação popular, pressupõe que os indivíduos aumentem o controle sobre suas vidas por meio da participação em grupos, com a finalidade de trans- formar a realidade social e política. Assim, as ações educativas deixam de ser apenas uma atividade a mais nos serviços de saúde, para ser algo que reorienta a diversidade das práticas realizadas ${ }^{(5)}$.

Estudos reforçam que atividades educativas com grupos são um importante dispositivo de promoção da saúde e como alternativa para as práticas assistenciais. É uma ótima possibilidade de área de atuação das equipes de Saúde da Família, principalmente do profissional enfermeiro ${ }^{(6-11)}$.

Diante dessas considerações, o objetivo do presente estudo consiste em identificar e analisar como as principais ações de Promoção da Saúde, estabelecidas pela Carta de Ottawa, estão sendo trabalhadas nas práticas educativas da Saúde da Família de um centro de saúde do Município de Florianópolis.

$\mathrm{Na}$ Carta de Ottawa, a educação em saúde destaca-se como princípio fundamental para a conquista da autonomia, participação, empoderamento e maior qualidade de vida da população. Ou seja, ao enfocar as estratégias de política públicas, reforço da ação comunitária, criação de habilidades pessoais, participação popular e reorientação dos serviços de saúde resgatam-se as ações de educação em saúde, que visam promover a saúde dos indivíduos e comunidades.

A realização deste estudo sustentou-se no argumento de que a priorização da educação em saúde no processo de trabalho da Saúde da Família pode ampliar a possibilidade de atuação e vinculação entre os profissionais (dentre eles o enfermeiro) e usuários; propiciar conhecimento para planejar e transformar as ações de saúde, visando a melhoria da qualidade de vida dos indivíduos; e tornar as práticas educativas mais efetivas, por meio da valorização da saúde e não da doença.

\section{METODOLOGIA}

Trata-se de uma pesquisa qualitativa articulada com o referencial metodológico de Paulo Freire. Consiste de três momentos dialética e interdisciplinarmente entrelaçados, que são: Investigação temática e levantamento dos temas geradores; Codificação e descodificação; Desvelamento crítico ou Problematização ${ }^{(12)}$.

Na etapa da Investigação temática, os temas geradores são extraídos da experiência das famílias, comunidades, profissionais. É a descoberta do universo vocabular, palavras ou temas extraídos do cotidiano das pessoas participantes nos círculos de cultura. $\mathrm{Na}$ 
Codificação e decodificação, os temas geradores são problematizados e contextualizados por meio do diálogo para uma visão crítica. O Desvelamento Crítico ou Problematização é a tomada de consciência da situação existencial quando se descobre os limites e as possibilidades; ocorre o processo de ação-reflexãoação para a superação das contradições da realidade vivida $^{(12)}$. O Itinerário de Pesquisa de Freire valoriza as fontes culturais e históricas dos indivíduos, que podem ser desveladas nos Círculos de Cultura.

O Círculo de Cultura é um termo criado por Freire, representado por um espaço dinâmico de aprendizagem e troca de conhecimento. Os sujeitos se reúnem no processo de educação para investigar temas de interesse do próprio grupo. Apresenta-se uma situação-problema real, que leva à reflexão da própria realidade, para, na sequência, decodificá-la e reconhecê-la ${ }^{(13)}$.

Como critério de seleção dos grupos, estabeleceu-se que estes deveriam ser previamente estruturados, com reuniões mensais e com a atuação de equipe multidisciplinar. Dessa forma, o estudo foi realizado no período de agosto a novembro de 2008, em três grupos de usuários portadores de hipertensão arterial e diabetes mellitus, em círculos de cultura com as respectivas equipes de Saúde da Família de um centro de saúde do Município de Florianópolis, no Estado de Santa Catarina.

Os Círculos de Cultura contaram com o envolvimento de dezenove profissionais incluindo os Agentes Comunitários de Saúde, enfermeiros, médicos, auxiliares/técnicos de enfermagem e odontólogos - não houve participação dos integrantes das equipes complementares: nutricionistas, psicólogos, assistentes sociais, que desenvolviam atividades nos grupos de hipertensos e diabéticos - além de 26 usuários participantes dos grupos.

Para a identificação dos temas geradores, foram lançados, durante os Círculos de Cultura, alguns questionamentos ligados a conceitos de saúde, doença, qualidade de vida, promoção da saúde, prevenção de doenças e, também, quanto ao objetivo do grupo, as dificuldades existentes, a metodologia utilizada e as possibilidades a serem trabalhadas. No total, foram 16 Círculos realizados durante os encontros dos grupos, que ocorreram nas instalações do centro de saúde, na comunidade e durante passeios previamente programados pelas equipes e usuários.

Os temas geradores destacados durante a investigação temática foram registrados por escrito, mediante um "diário de campo", no qual, em cada Círculo de Cultura, um dos pesquisadores conduzia o diálogo sobre o tema em estudo. Ao mesmo tempo, outro pesquisador colaborava registrando o produto do debate em diário de campo. Todas as anotações e transcrições foram arquivadas em pastas digitais para as etapas posteriores da codificação, descodificação e desvelamento crítico.

A etapa de codificação e descodificação dos dados ocorreu por meio de leitura cuidadosa das informações obtidas nos Círculos de Cultura. Foram identificadas as temáticas significativas de cada atividade realizada, relacionando-as com o tema do estudo, ou seja, as cinco ações de Promoção da Saúde propostas pela Carta de Ottawa: políticas públicas saudáveis, criação de ambientes favoráveis, reforço da ação comunitária, criação de habilidades pessoais e reorientação dos serviços de saúde ${ }^{(1)}$. Posteriormente, os dados destacados nortearam a reflexão com os sujeitos participantes, com o objetivo de descodificar e devolver os temas geradores identificados pelo grupo.

A pesquisa atendeu os requisitos éticos contidos na Resolução 196/1996 ${ }^{(14)}$, e foi aprovada pelo Comitê de Ética da Universidade Federal de Santa Catarina (UFSC), sob o Protocolo n. 158/08. O anonimato dos sujeitos foi garantido e o nome do centro de saúde não foi mencionado. Antes de cada Círculo de Cultura, foi realizado o esclarecimento da pesquisa e solicitada a assinatura do termo de consentimento dos profissionais das equipes da ESF, ACS e usuários participantes do estudo. A adesão dos participantes foi voluntária, sem número limitado.

\section{RESULTADOS}

Os temas geradores codificados e descodificados estavam ligados e foram categorizadas nas cinco estratégias da Promoção da Saúde, referidas na Carta de Ottawa ${ }^{(1)}$ e especificadas a seguir.

\section{Elaboração e Implementação de Políticas Públicas Saudáveis}

A temática investigada relacionada a esta estratégia de Promoção da Saúde foram as limitações intersetoriais e interdisciplinares para o desenvolvimento das práticas educativas na Saúde da Família.

O centro de saúde tem representação profissional no Conselho Local, assim como no Conselho Comunitário. As reuniões eram realizadas mensalmente, 
com a participação de algumas lideranças afiliadas da comunidade. A dificuldade de concretização imediata de atenção às necessidades apresentadas nas reuniões comunitárias tem contribuído para desmobilização dos diversos representantes setoriais de educação, saúde, igrejas e moradores que participam dos Conselhos. Avaliou-se que entraves políticos e administrativos têm contribuído para a morosidade no atendimento das questões pontuadas e fundamentais para a saúde da comunidade. Os Círculos de Cultura revelaram que neste microespaço reproduzem-se as tradicionais disputas de poder político, corporativo e econômico, frequentes em outras instâncias governamentais ou não. Este fato fragiliza as relações intersetoriais e interdisciplinares limitando a concretização imediata das ações que pudessem superar diversos determinantes do processo saúde e doença da população local.

A interdisciplinaridade acontecia de forma iniciante e positiva, agregando escolas, organizações religiosas e comunitárias não governamentais. Aconteceram periodicamente reuniões com professores de dança, educadores físicos e moradores voluntários da comunidade local. Nestes encontros, desenvolveram-se atividades lúdicas, físicas e de confecção de material artesanal, organizadas pela enfermeira, na Unidade de Saúde. Da mesma forma, estudantes do Curso de Agronomia de duas universidades públicas apoiam o desenvolvimento de atividades agrícola comunitária em horta no centro de saúde. Este fato firma elos interdisciplinares, possibilitando a criação de vínculos entre pessoas de diferentes setores da comunidade, tais como a escola, o centro de saúde, os grupos comunitários de artesanato e estudantes e profissionais das universidades que atuam na área.

\section{Criação de Ambientes Favoráveis à Saúde}

Os temas geradores relacionados com esta ação emergiram das atividades que estavam sendo desenvolvidas no centro de saúde e na comunidade, as quais visavam a criação de ambientes que pudessem contribuir para a maior qualidade de vida da população. A comunidade local demonstrou desejo de suprir necessidade de espaços laborais, lazer, atividade física e educativa para nutrição saudável. Além disso, percebeu-se a necessidade de maior quantidade e qualidade dos serviços de transporte público local. O centro de saúde, juntamente com a escola, apresentou as principais opções de reivindicação e acolhimento das necessidades manifestadas pela comunidade. Es- tes espaços são entendidos como fortalezas capazes de alavancar a criação de novos ambientes que promovam saúde e educação, além de suas atividades de rotina. Assim sendo, os profissionais do centro de saúde exerciam, além da sua prática cotidiana, a função de promover a criação de ambientes saudáveis, como estimular a participação da população nos espaços de lazer, praças, parques, quadras de esporte, atividades artísticas e culturais. Para suprir tais carências, muitas vezes a população recorre ao centro de saúde, em busca, além da resolutividade de uma situação de saúde-doença ou auxílio de seus problemas sociais, também à procura de entretenimento, de diálogo e de afastamento da monotonia diária.

\section{Reforço da Ação Comunitária}

Em relação a esta estratégia, emergiu a questão da necessidade de incentivo e investimento na formação de agentes educativos para fomento da participação comunitária, corresponsabilidade individual e coletiva para o desenvolvimento das práticas educativas dos indivíduos e comunidade. Esta é uma estratégia para promoção da saúde contida na Carta de Ottawa ${ }^{(1)}$. No centro de saúde citado nesta experiência, percebe-se alguma articulação e participação na estratégia para reforçar a ação comunitária local. Trata-se, ainda, de atitudes pontuais que necessitam ser intensificadas para garantir a maior participação, incentivo, estímulo, autonomia, assim como dar respostas concretas para algumas demandas imediatas, capazes de produzir motivação para novas conquistas.

\section{Desenvolvimento de Habilidades Pessoais}

Dos questionamentos referentes à qualidade de vida, conceitos de saúde e doença, apareceram os temas geradores: Saúde entendida como ausência de doença e dúvidas e desconhecimento sobre diabetes e hipertensão.

Apesar do conceito de saúde ter sofrido alterações ao longo do tempo e de ser bastante discutido entre os profissionais de saúde, ainda se faz muito presente, no cotidiano dos usuários, a saúde como ausência de doença, tendo o médico como principal agente na atuação da prevenção de doenças, na cura da patologia e na "promoção" da saúde.

Questionados sobre o processo saúde-doença, especialmente com relação às doenças crônicas, os sujeitos do estudo manifestaram pouca compreensão, 
e, muitas vezes, consideram-se "curados" do diabetes ou da hipertensão. E os que ainda não conseguiram a "cura", procuram-na arduamente, por meios científicos ou populares, visando eliminar a patologia de seu organismo.

Somado a isso, houve muitos relatos no tocante ao tratamento e à sintomatologia, demonstrando insegurança e dúvidas. Porém, afirmaram querer aprender "coisas novas" em relação ao cuidado com sua saúde e o manejo com a doença. Neste sentido, os Círculos de Cultura motivaram as equipes de Saúde da Família a incluir as estratégias e os princípios da promoção da saúde contidos na Carta de Ottawa nas suas práticas educativas.

\section{Reorientação dos Serviços de Saúde}

Nesta ação, foram relacionados os temas geradores: Dificuldades para compreensão e desenvolvimento dos grupos em Saúde da Família; Necessidade de maior discussão/compreensão sobre Promoção da Saúde; Sobrecarga de trabalho e; Importância do vínculo entre profissional e usuário.

As reflexões oriundas destas temáticas emergiram da percepção de que deveria ocorrer um grande esforço dos profissionais em realizar atividades, principalmente agradáveis e atrativas, para os usuários hipertensos e diabéticos. Os ACS promovem, em cada mês, uma grande divulgação para convidar a população a comparecer ao grupo, e a equipe de saúde como um todo tenta planejar as atividades a serem desenvolvidas a partir da necessidade relatada pelos usuários.

Porém, os trabalhadores demonstram grande frustração por muitos usuários não participarem dos grupos e outros não serem frequentadores assíduos, o que provoca discussões quanto à sua efetividade $\mathrm{e}$ uma necessidade de reavaliação da metodologia. Em muitos relatos e reflexões, usuários e profissionais mostram incertezas e contradições quanto ao que consideram ser o objetivo do grupo. Os primeiros referem que o grupo é um momento de lazer, descontração e entretenimento. E os profissionais demonstram inseguranças, dúvidas e concepções diferentes em relação às atividades a serem desenvolvidas nos encontros.

Aliado a isso, a sobrecarga de trabalho dos profissionais, devido à grande demanda por atendimento individual nos consultórios, também foi mencionada como um problema para dedicação e disponibilidade de tempo para o planejamento e preparação destas atividades.

\section{DISCUSSÃO}

De acordo com o nosso estudo, identificamos diversas fragilidades inerentes à implementação das estratégias de promoção da saúde na realidade de uma unidade de atenção básica. Avaliamos que o modelo assistencial é primordialmente centrado na atenção à demanda de ações curativas e individuais. Entretanto, notamos algumas atitudes positivas dos profissionais em direção às mudanças deste modelo, com possibilidade de desenvolvimento de ações de promoção da saúde, de acordo com os princípios da Carta de Ottawa. Estas propostas, mesmo incipientes, constituem-se em espaços modelares para estímulo ao envolvimento dos profissionais e população. Identificamos a necessidade de investimentos na política de formação profissional e de educação permanente, incluindo a promoção da saúde, como uma política pública capaz de produzir transformação no modelo vigente. Ao mesmo tempo estimulou-se a população encorajando-a em atividades de saúde e empoderando-as para melhorar a sua condição de vida. Seria necessário investir no conhecimento do ser humano local, identificando suas circunstâncias pessoais e coletivas, culturais, valores e tradições. Com isso, estabelecer uma maior aproximação no sentido de contar com as pessoas e comunidade, motivando-os ao cuidado de si e do outro.

Estrategicamente, os grupos de educação em saúde constituem-se em espaços potencialmente privilegiados para o empoderamento individual e coletivo. A valorização da participação da população no controle social dos serviços recoloca o trabalho educativo realizado em grupos em novo aspecto, possibilitando organizar estratégias coletivas para o enfrentamento dos determinantes do processo saúde-doença ${ }^{(15)}$. Por meio deles, pode ocorrer o desenvolvimento pessoal e social, com divulgação de informação, diálogo e intensificação das habilidades vitais. É essencial capacitar as pessoas para aprender durante toda a vida, preparando-as para as diversas fases da existência, o que inclui o enfrentamento das doenças crônicas e causas externas $^{(1,16)}$.

Nessa perspectiva, a Promoção da Saúde vai além dos cuidados de saúde. Ela coloca a saúde na agenda de prioridades dos políticos e gestores de todos os níveis e setores, que devem tomar consciência de suas decisões e responsabilidades; sugere ações legislativas, fiscais e organizacionais visando a diminuição das desigualdades sociais e a melhoria da qualidade de vida da população. Propõe, também, um diálogo 
interdisciplinar, ações intersetoriais e a participação das comunidades envolvidas para a formulação de políticas públicas e sua ação sobre o setor saúde ${ }^{(1,16)}$. A promoção de saúde trabalha por meio de ações comunitárias concretas e efetivas visando a melhoria das condições de saúde, ao priorizar a implementação de ações e recursos existentes na comunidade que possam intensificar a autoajuda e o apoio social necessários ao desenvolvimento da participação popular nos assuntos de saúde, o empoderamento comunitário. Isto requer um total e contínuo acesso à informação, às oportunidades de aprendizado para os assuntos de saúde, assim como apoio financeiro adequado da população comunitária local ${ }^{(1,16)}$.

Para tanto, as abordagens educativas devem valorizar a criação de espaços públicos (rodas e grupos de discussão, como os de hipertensos e diabéticos, colegiados, gestores), que promovam a participação dos indivíduos e da coletividade na identificação e na análise crítica de seus problemas, objetivando a elaboração de estratégias de ação que busquem a transformação da realidade ${ }^{(17)}$. As ações de promoção devem aproximar as relações entre a população e seu meio ambiente, que constituem a base para uma abordagem socioecológica da saúde. A proteção do meio ambiente, a mudança nos modos de vida, de trabalho e de lazer têm um significativo impacto sobre a saúde ${ }^{(1,16)}$.

Dessa forma, para que se possa capacitar as pessoas a aprender por sua própria experiência de vida e prepará-las para todos os estágios ${ }^{(18)}$, além de promover o desenvolvimento pessoal e social por meio da divulgação de informação, educação para a saúde e intensificação das habilidades vitais ${ }^{(1)}$, devese implementar práticas que tenham como meta o desenvolvimento de habilidades pessoais. Os profissionais devem estabelecer vínculos e criar laços de corresponsabilidade com os usuários que irão decidir o que é bom para si, segundo suas próprias crenças, valores, expectativas e necessidades ${ }^{(18-19)}$.

A responsabilidade pela promoção da saúde nos serviços de saúde deve ser compartilhada entre indivíduos, comunidade, grupos, profissionais da saúde, instituições que prestam serviços de saúde e governos; deve voltar-se na direção de um enfoque na saúde e não na doença, que apontem para a integralidade das ações de saúde ${ }^{(1,16)}$. No entanto, a reorientação dos serviços de saúde requer um esforço maior de pesquisa em saúde, assim como de mudanças na educação e no ensino dos profissionais da área da saúde.
Deste modo, as ações de Promoção da Saúde são resultantes de um complexo processo que envolve o fortalecimento das capacidades individuais e coletivas atuando sobre múltiplas dimensões: por um lado, intervenções de âmbito global do Estado e, por outro, a singularidade e autonomia dos sujeitos ${ }^{(20)}$.

\section{CONSIDERAÇÕES FINAIS}

Por meio da metodologia de Paulo Freire, foi possível realizar um diálogo crítico com os sujeitos participantes de Círculos de Cultura nos microespaços político e social, nas atividades de grupos, promovendo ações concretas que contribuíram para transformação daquela realidade. A Promoção da Saúde foi contemplada em discussão nos grupos de portadores de hipertensão arterial e diabetes mellitus, superando a visão curativa focada na recepção dos medicamentos específicos. Perceberam-se avanços na compreensão destes usuários quanto ao seu processo saúde-doença, identificando sua determinação e optando por novas possibilidades de ser saudável, tais como opções de lazer, alimentação saudável e oportunidade de atividades físicas que contribuam na sua qualidade de vida.

Porém, a incorporação das ações de Promoção de Saúde ainda está distante da prática concreta dos profissionais, especialmente, devido ao modelo de atenção à saúde, que tem como base de sustentação um modelo biomédico individual e curativo. É preciso que os profissionais de saúde compreendam que a Promoção constitui-se em campo amplo e complexo, que envolve diversas estratégias de intervenção, com atuação desde a clínica até o contexto dos determinantes sociais e políticos do processo saúde-doença. Na realidade, percebeu-se a importância de estimular o empoderamento dos múltiplos atores sociais.

Como recomendação, salienta-se que o trabalho desenvolvido pelas equipes de Saúde da Família seja interdisciplinar e dialógico, para que os usuários expressem suas reais necessidades de saúde. Além disso, sugere-se a realização de trabalhos que identifiquem as dificuldades e facilidades para concretizar a prática educativa nos serviços, no sentido de revelar o valioso instrumento que esta ação possibilita para a melhoria da qualidade de vida da população.

\section{REFERÊNCIAS}

1. Organização Mundial de Saúde. Carta de Ottawa, aprovada na I Conferência Internacional sobre Promoção da Saúde em 1986. 1986 [acesso em 17 ago 
2010]. Disponível: http://tiny.cc/9jd8u

2. Sucupira AC. Marco conceitual da promoção da saúde no PSF. Sanare. 2003;4(1):11-14.

3. Lefèvre F, Cavalcanti AMC. Promoção de saúde: a negação da negação. Rio de Janeiro (RJ): Vieira \& Lent; 2004.

4. Oliveira DL. A "nova” saúde pública e a promoção da saúde via educação: entre a tradição e a inovação. Rev Latino-Am Enfermagem. 2005;13(3):423-31.

5. Vasconcelos EM. Educação popular e atenção à saúde da família. 2a ed. São Paulo: Hucitec; 1999.

6. Silva ALAC, Munari DB, Lima FV, Silva WO. Atividades grupais em saúde coletiva: características, possibilidades e limites. Rev Enferm UERJ. 2003;11(1):18-24.

7. Silva DGVS, Francioni FF, Natividade MSL, Azevedo M, Sandoval RCB, Di'Lourenzo VM. Grupos como possibilidade para desenvolver educação em saúde. Texto \& Contexto Enferm. 2003;12(1):97-103.

8. Torres HC, Hortale VA, Schall V. A experiência de jogos em grupos operativos na educação em saúde para diabéticos. Cad Saúde Pública. 2003;29(4):1039-47.

9. Alves, LHS. Grupo de promoção da saúde na Estratégia Saúde da Família (ESF): a visão dos profissionais e dos usuários [dissertação]. Florianópolis (SC): Universidade Federal de Santa Catarina; 2010.

10. Maffacciolli R, Lopes MJM. Educação em saúde: a orientação alimentar através de atividades de grupo. Acta Paul Enferm. 2005;18(4):439-45.

11. Silva MA, Oliveira AGB, Mandú ENT, Marcon SR. Enfermeiro e grupos em PSF: possibilidade para participação social. Cogitare Enferm. 2006;11(2):143-9.

12. Freire P. Pedagogia do oprimido. $17^{\mathrm{a}}$ ed. Rio de Janeiro: Paz e Terra; 1987.

13. Freire, P. Pedagogia da esperança. Rio de Janeiro: Paz e Terra; 1992.

14 Ministério da Saúde (BR). Conselho Nacional de Saúde. Diretrizes e normas regulamentadoras de pesquisa envolvendo seres humanos. Resolução n. 196, de 10 de outubro de 1996. Brasília; 1996.

15. Basso JF. Reflexões de um grupo de gestantes e acompanhantes sobre a participação ativa no parto e nascimento [dissertação]. Florianópolis (SC): Universidade Federal de Santa Catarina; 2007.

16. Heidmann ITSB, Almeida MCP, Boehs AE, Wosny $\mathrm{AM}$, Monticelli M. Promoção à saúde: trajetória histórica de suas concepções. Texto \& Contexto Enferm. 2006;15(2):352-358.

17. Carvalho SR. Os múltiplos sentidos da categoria "empowerment" no projeto de promoção à saúde. Cad. Saúde Pública. 2004;20(4):1088-95.

18. Heidemann ITSB. A promoção da saúde e a concepção dialógica de Freire: possibilidades de sua inserção e limites no processo de trabalho das equipes de Saúde da Família [tese]. Ribeirão Preto (SP): Universidade de São Paulo; 2006.

19. Ministério da Saúde (BR). Secretaria de Assistência à Saúde. Coordenação de Saúde da Comunidade. Saúde da Família: uma estratégia para a reorientação do modelo assistencial. Brasília: Ministério da Saúde; 1997.

20. Santos SMR, Jesus MCP, Amaral AMM, Costa DMN, Arcanjo RA. Consulta de enfermagem no contexto da atenção básica de saúde, Juiz de Fora, MG. Texto \& Contexto Enferm. 2008;17(1):124-30. 